\title{
Fractional-Order Discrete-Time Laguerre Filters: A New Tool for Modeling and Stability Analysis of Fractional-Order LTI SISO Systems
}

\author{
Rafał Stanisławski and Krzysztof J. Latawiec \\ Department of Electrical, Control and Computer Engineering, Opole University of Technology, Prószkowska 76, 45-758 Opole, Poland \\ Correspondence should be addressed to Rafał Stanisławski; r.stanislawski@po.opole.pl
}

Received 13 April 2016; Accepted 13 June 2016

Academic Editor: Chris Goodrich

Copyright (C) 2016 R. Stanisławski and K. J. Latawiec. This is an open access article distributed under the Creative Commons Attribution License, which permits unrestricted use, distribution, and reproduction in any medium, provided the original work is properly cited.

\begin{abstract}
This paper presents new results on modeling and analysis of dynamics of fractional-order discrete-time linear time-invariant single-input single-output (LTI SISO) systems by means of new, two-layer, "fractional-order discrete-time Laguerre filters." It is interesting that the fractionality of the filters at the upper system dynamics layer is directly projected from the lower Laguerre-based approximation layer for the Grünwald-Letnikov difference. A new stability criterion for discrete-time fractional-order Laguerrebased LTI SISO systems is introduced and supplemented with a stability preservation analysis. Both the stability criterion and the stability preservation analysis bring up rather surprising results, which is illustrated with simulation examples.
\end{abstract}

\section{Introduction}

It is well known that orthonormal basis functions (OBF) can be a good alternative to, for example, state space or ARX models when solving the problems in modeling and identification of some classes of "regular" or integer-order dynamical systems, both linear [1-3] and nonlinear ones [4,5]. Recently, the OBF machinery has increasingly been used in modeling and identification tasks for noninteger or fractional-order systems [6-9], a broad research field attracting a huge interest both from the academia and industrial environments [10-17]. In this paper we limit ourselves to discrete-time fractionalorder systems. There are two main research directions for potential use of OBF, in particular Laguerre functions, in the fractional-order modeling context, namely, Laguerrebased approximation of fractional-order Grünwald-Letnikov (GL) derivatives $[8,9]$ and Laguerre-based modeling of fractional-order dynamical systems $[18,19]$. We pursue both research directions, employing discrete-time Laguerre filters to effectively model both the GL fractional-order difference (FD) $[8,9]$ and dynamics of a discrete-time fractional-order plant to be covered here. Such a two-layer Laguerre modeling idea is a new contribution to modeling of linear discrete-time fractional-order systems. Our new Laguerre-based approximators to FD, called FLD and FFLD $[8,9]$, follow the lines of the introduction of the "classical" (truncated or) finite FD, that is, FFD.

The FD and FFD [20] are often used in the context of modeling of discrete-time fractional-order LTI state space systems [14, 20-22]. It is the asymptotic stability that is considered the most important aspect of an analysis of fractional-order LTI systems [23-28]. As for continuous-time systems, the celebrated Matignon's stability criterion [29] has established a simple argument condition for eigenvalues of a state matrix. It is only recently that a thorough asymptotic stability analysis has been made for discrete-time fractionalorder LTI state space systems $[30,31]$ and the long-awaited stability criterion has been introduced for such systems [31]. That criterion will be reformulated here for LTI systems described by a new fractional-order model based on "fractionalized" Laguerre filters.

In this paper, a new concept of "fractional-order discrete-time Laguerre filters" is introduced for modeling of fractional-order LTI SISO systems and a new analytical stability criterion is announced. Also, the stability preservation problem is solved. 
The remainder of this paper is organized as follows. Fundamentals of the regular OBF system description, in particular the Laguerre filters, are recalled in Section 2, with an important factorization of the Laguerre transfer functions presented. The first significant result of this paper is provided in a unified framework in Section 3, where a new term of "fractional-order discrete-time Laguerre filters" is coined, supported with a series of preliminary outcomes preparing for the main result of Section 4. That section provides a new general criterion for the asymptotic stability of discretetime LTI SISO fractional-order Laguerre systems, being a nice supplementation of the criterion of [31]. The criterion is followed by a stability preservation analysis in Section 5 . The parameter estimation problem for fractional-order Laguerre systems is outlined in Section 6 and the reorthonormalization issue is addressed in Section 7. Conclusions of Section 8 summarize the achievements of the paper.

\section{Regular OBF System Description}

An output of a "regular" (or integer-order) discrete-time OBF-modeled dynamical system or shortly OBF system can be described as

$$
y(t)=\sum_{i=1}^{K} C_{i} L_{i}\left(q^{-1}\right) u(t)
$$

where $q^{-1}$ is the backward shift operator, $u(t)$ and $y(t)$ are the system input and output, respectively, at discrete-time $t=$ $0,1, \ldots$, and $L_{i}\left(z^{-1}\right)$ and $C_{i}, i=1, \ldots, K$, are orthonormal transfer functions and weighting parameters, respectively. For discrete-time Laguerre filters there is

$$
L_{i}\left(z^{-1}\right)=\frac{k z^{-1}}{1-P z^{-1}}\left(\frac{-P+z^{-1}}{1-P z^{-1}}\right)^{i-1}, \quad i=1, \ldots, K
$$

with $\underline{k}=\sqrt{1-P^{2}}$ and $P$ being a dominant pole. We proceed with the practically justified case of $1>P>$ 0 . The unknown parameters $C_{i}, i=1, \ldots, K$, can be easily estimated via, for example, Recursive Least Squares (RLS) or Least Mean Squares (LMS) algorithms using the linear regression formalism. We discriminate between the parameters of the Laguerre model of a dynamical system $\left(P, K, \underline{k}, C_{i}, i=1, \ldots, K\right)$ and those for the Laguerre-based fractional-order difference $\left(p, M, k, c_{l}, l=1, \ldots, M\right)$ as in $[8,9]$.

Remark 1. Note that we do not need to account for the sampling period $T$ in $\underline{k}$ as it will be indirectly included in the estimated parameters $C_{i}, i=1, \ldots, K$.

Remark 2. Note that pursuing an optimal Laguerre pole $P_{\mathrm{opt}}$ has been well established [2, 32-34].

Depending on the time-domain or $z$-domain contexts, we will interchangeably use the notations $L_{i}\left(q^{-1}\right)$ or $L_{i}\left(z^{-1}\right)$, respectively. The same manner will be applied to other functions of $q^{-1}$ or $z^{-1}$.
A crucial factorization is recalled; that is, (2) is rewritten as $[19,35]$

$$
L_{i}\left(z^{-1}\right)=G_{L}\left(z^{-1}\right)\left(G_{R}\left(z^{-1}\right)-P\right)^{i-1}, \quad i=1, \ldots, K
$$

with

$$
\begin{aligned}
& G_{L}\left(z^{-1}\right)=\frac{\underline{k} z^{-1}}{1-P z^{-1}}, \\
& G_{R}\left(z^{-1}\right)=\frac{\underline{k}^{2} z^{-1}}{1-P z^{-1}}=\underline{k} G_{L}\left(z^{-1}\right)
\end{aligned}
$$

and the filter outputs obtained as $y_{L}(t)=G_{L}\left(q^{-1}\right) u(t)$ and $y_{R}^{i}(t)=G_{R}\left(q^{-1}\right) U_{i}(t), i=1, \ldots, K-1$, with

$$
U_{i}(t)= \begin{cases}y_{L}(t), & i=1 \\ y_{R}^{i-1}(t)-P U_{i-1}(t), & i=2, \ldots, K .\end{cases}
$$

The filters $G_{L}$ and $G_{R}$ of (4) can be described in terms of the backward differences

$$
\begin{aligned}
& G_{L}: \Delta y_{L}(t)=(P-1) y_{L}(t) q^{-1}+\underline{k} u(t) q^{-1}, \\
& G_{R}: \Delta y_{R}^{i}(t)=(P-1) y_{R}^{i}(t) q^{-1}+\underline{k}^{2} U_{i}(t) q^{-1},
\end{aligned}
$$

where $\Delta y_{L}(t)=y_{L}(t)-y_{L}(t-1)$ and $\Delta y_{R}^{i}(t)=y_{R}^{i}(t)-y_{R}^{i}(t-$ 1), $i=1, \ldots, K$.

\section{FD/FFD/LD/FLD/CFLD/FFLD-Based Laguerre Systems: Unified Framework}

We refer to the Grünwald-Letnikov (GL) discrete-time fractional-order difference (FD) and its Laguerre-based equivalents LD and CFLD $[8,9]$ as well as to their approximators FFD [20], FLD, and FFLD [8, 9]. We strongly advocate the use of the FFLD approximator to FD, being an effective combination of FFD and FLD [9]. In FFLD, the FFD share is to precisely model the high-frequency properties of FD whereas FD's long “tale" is excellently modeled by FLD [9]. We will shortly write "FD/FFD/LD/FLD/CFLD/FFLD-based Laguerre system" instead of "Laguerre model of a fractionalorder system based on FD/FFD/LD/FLD/CFLD/FFLD.”

Here is the first new result of this paper, that is, the introduction of a concept of "fractional-order discrete-time Laguerre filters."

Theorem 3. Consider the FD/FFD/LD/FLD/CFLD/FFLD fractional-order differences $[8,9]$ uniformly described as

$$
\begin{aligned}
\Delta^{\alpha} x(t)= & x(t)+\sum_{j=1}^{J} P_{j}(\alpha) x(t-j) \\
& +\sum_{l=1}^{M} c_{l} L_{l}\left(q^{-1}\right) x(t-\bar{J}) \\
= & x(t)+X(t, J, M), \quad t=0,1, \ldots
\end{aligned}
$$

with $P_{j}(\alpha), j=1, \ldots, J, J=\min (t, \bar{J})$ and $\bar{J}$ is the upper bound for $j$ whent $>\bar{J}[9,20]$, and $L_{l}\left(z^{-1}\right), l=1, \ldots, M$, being 
the Laguerre filters and the particular differences specified as

(i) FD for $\bar{J} \rightarrow \infty$ and $M=0$,

(ii) FFD for $\bar{J}<\infty$ and $M=0$,

(iii) $L D$ for $\bar{J}=0, M \rightarrow \infty$, and $c_{l}$ calculated as in Theorem 1 of $[8,9]$,

(iv) FLD for $\bar{J}=0, M<\infty$, and $c_{l}$ calculated as in Theorem 1 of $[8,9]$,

(v) CFLD for $0<\bar{J}<\infty, M \rightarrow \infty$, and c calculated as in Theorem 2 of $[8,9]$,

(vi) FFLD for $0<\bar{J} M<\infty$ and $c_{l}$ calculated as in Theorem 2 of $[8,9]$.

Then FD/FFD/LD/FLD/CFLD/FFLD-based fractionalorder LTI SISO Laguerre system can be described as

$$
y(t)=\sum_{i=1}^{K} C_{i} L_{i}^{f}\left(q^{-1}\right) u(t),
$$

where $u(t)$ and $y(t)$ are the system input and output, respectively, $C_{i}, i=1, \ldots, K$, are the unknown parameters, and the "fractional-order discrete-time Laguerre filters" are as follows:

$$
\begin{aligned}
L_{i}^{f}\left(q^{-1}\right)=G_{L}^{f}\left(q^{-1}\right)\left(G_{R}^{f}\left(q^{-1}\right)-P\right)^{i-1}, & \\
& i=1, \ldots, K
\end{aligned}
$$

with $P$ being the dominant Laguerre pole and

$$
\begin{aligned}
& G_{L}^{f}\left(q^{-1}\right)=\frac{\underline{k} q^{-1}}{(1-P) q^{-1}+F\left(q^{-1}\right)}, \\
& G_{R}^{f}\left(q^{-1}\right)=\underline{k} G_{L}^{f}\left(q^{-1}\right)
\end{aligned}
$$

with $\underline{k}=\sqrt{1-P^{2}}$ and

$$
F\left(q^{-1}\right)=1+\sum_{j=1}^{\bar{J}} P_{j}(\alpha) q^{-j}+\sum_{l=1}^{M} c_{l} L_{l}\left(q^{-1}\right) q^{-\bar{J}}
$$

pertaining to specifications (i) to (vi) for the particular differences.

Proof. Accounting for (6) on the one hand and (8) on the other hand (compare [9]), the fraction-formalized filters $G_{L}^{f}\left(z^{-1}\right)$ and $G_{R}^{f}\left(z^{-1}\right)$ can now be described as

$$
\begin{aligned}
G_{L}^{f}: \Delta^{\alpha} y_{L}(t) & =(P-1) y_{L}^{f}(t) q^{-1}+\underline{k} u(t) q^{-1} \\
\Delta^{\alpha} y_{L}(t) & =y_{L}^{f}(t)+Y_{L}(t, J, M), \\
G_{R}^{f}: \Delta^{\alpha} y_{R}^{i}(t) & =(P-1) y_{R}^{i, f}(t) q^{-1}+\underline{k}^{2} U_{i}^{f}(t) q^{-1} \\
\Delta^{\alpha} y_{R}^{i}(t) & =y_{R}^{i, f}(t)+Y_{R}^{i}(t, J, M), \\
& i=1, \ldots, K-1
\end{aligned}
$$

with

$$
\begin{aligned}
Y_{L}(t, J, M)= & \sum_{j=1}^{J} P_{j}(\alpha) y_{L}^{f}(t-j) \\
& +\sum_{l=1}^{M} c_{l} L_{l}\left(q^{-1}\right) y_{L}^{f}(t-\bar{J}), \\
Y_{R}^{i}(t, J, M)= & \sum_{j=1}^{J} P_{j}(\alpha) y_{R}^{i, f}(t-j) \\
& +\sum_{l=1}^{M} c_{l} L_{l}\left(q^{-1}\right) y_{R}^{i, f}(t-\bar{J})
\end{aligned}
$$

and $U_{i}^{f}(t), i=1, \ldots, K$, is computed as

$$
U_{i}^{f}(t)= \begin{cases}y_{L}^{f}(t), & i=1 \\ y_{R}^{i-1, f}(t)-P U_{i-1}^{f}(t), & i=2, \ldots, K .\end{cases}
$$

Finally, the outputs from the two fractional filters are

$$
\begin{aligned}
G_{L}^{f}: y_{L}^{f}(t)= & (P-1) y_{L}^{f}(t) q^{-1}+\underline{k} u(t) q^{-1} \\
& -Y_{L}(t, J, M), \\
G_{R}^{f}: y_{R}^{i, f}(t)= & (P-1) y_{R}^{i, f}(t) q^{-1}+\underline{k}^{2} U_{i}^{f}(t) q^{-1} \\
& -Y_{R}^{i}(t, J, M)
\end{aligned}
$$

with $U_{i}^{f}(t), i=1, \ldots, K-1$, calculated as in (17). Now, from (18) and (19) we can easily obtain transfer functions (11) and (12), respectively.

Conclusively, the output from the FD/FFD/LD/FLD/ CFLD/FFLD-based fractional Laguerre system is

$$
y(t)=\sum_{i=1}^{K} C_{i} U_{i}^{f}(t)
$$

where $U_{i}^{f}(t)=L_{i}^{f}\left(q^{-1}\right) u(t), i=1, \ldots, K$, and $L_{i}^{f}\left(q^{-1}\right)$ are given as in (10).

An outstanding value of Theorem 3 is at least threefold. Firstly, a new concept of "fractional-order discrete-time Laguerre filters" is introduced. Secondly, the factorization of the fractional-order Laguerre filters holds true in the same way as for the regular (nonfractional) ones, with (10) being still valid. This means that the block diagram for the fractional Laguerre system is identical with that for the regular one, with $G_{L}^{f}$ and $G_{R}^{f}$ substituted for $G_{L}$ and $G_{R}$, respectively. This provides a nice technical tool for modeling in, for example, Matlab. Thirdly, the whole calculation process for the model output of the fractional Laguerre system is quite similar to that for the regular Laguerre system, with an additional fractional component $F\left(q^{-1}\right)$ involved in the former case (see (11)). 
Remark 4. Note that FFLD as in (7) and (8) can be considered as one general unified model from which all the other considered models can be obtained according to specifications (i) through (v), with FFLD itself covering specification (vi).

Remark 5. Note that the whole "fractionality" of the otherwise (two-layer) fractional-order Laguerre system is contained in the (lower-layer) transfer function $F\left(z^{-1}\right)$. Also note that for the FD-based fractional Laguerre system we have the familiar GL transfer function $F\left(z^{-1}\right)=\left(1-z^{-1}\right)^{\alpha}$ (compare [30]). Of course, for $\alpha=1$ we arrive at the classical Laguerre filters $G_{L}$ and $G_{R}$.

Remark 6. Let us emphasize that the transfer function $F\left(z^{-1}\right)$ is related to the function $\Psi(\varphi)$ (or $\Psi(i \varphi))$ introduced in [30] in the asymptotic stability analysis of fractional-order discretetime state space systems. In fact, we have

$$
\Psi(\varphi)=\left.F\left(z^{-1}\right)\right|_{z=e^{i \varphi}}
$$

with $i$ being the imaginary unit and $\varphi=\arg z, 0 \leq \varphi \leq 2 \pi$.

Remark 7. Possible accounting for the sampling period $T$ in FD/FFD/LD/FLD/CFLD/FFLD-based Laguerre systems when transferring from a continuous-time (RiemannLiouville/Grünwald-Letnikov) derivative to the discrete-time difference results in the substitutions $(P-1) \rightarrow T^{\alpha}(P-1)$ and $k \rightarrow T^{\alpha} k$. This finally leads to the substitution $F\left(q^{-1}\right) \rightarrow F\left(q^{-1}\right) / T^{\alpha}$ in (11).

Remark 8. In addition to computational simplicity, the Laguerre-based difference is attractive also in that it can model both well-damped $(\alpha \in(0,1))$ and oscillatory behaviors $(\alpha \in$ $(1,2))$, without referring to, for example, the Kautz filters in the latter case.

An exemplary block diagram of the output calculation process for the FLD-based fractional Laguerre system is presented in Figure 1, with the "internal" (or lower-layer) FLD being additionally zoomed.

\section{Stability Results for Fractional-Order Laguerre Systems}

4.1. Stability of FD/FFD/LD/FLD/CFLD/FFLD-Based Laguerre Systems: A Unified Framework. We adopt the stability results of $[30,31]$ to the Laguerre-based fractional systems to obtain an original outcome which is the main result of this paper.

Theorem 9. Consider the FD/FFD/LD/FLD/CFLD/FFLDbased Laguerre system described by (7) to (20) with $Y_{L}(t, J, M)$ and $Y_{R}^{i}(t, J, M), i=1, \ldots, K-1$, computed from (15) and (16) under respective specifications ( $i)$ to (vi) using $y_{L}^{f}(t)$ and $y_{R}^{i, f}(t), i=1, \ldots, K-1$, as in (18) and (19), respectively. The system is asymptotically stable if and only if

$$
P-1<\Psi(0), \quad \forall \alpha \in(0,2) \wedge P>0,
$$

where $\Psi(0)=\left.\Psi(\varphi)\right|_{\varphi=0}$ and $\Psi(\varphi)$ defined as in (21), with

(i) $\Psi(0)=0$ for the FD/LD/CFLD-based systems,

(ii) $\Psi(0)=1+\sum_{j=1}^{\bar{J}} P_{j}(\alpha)$ for the FFD-based system,

(iii) $\Psi(0)=1+(k /(1-p)) \sum_{l=1}^{M} c_{l}$, with $c_{l}, l=1, \ldots, M$, as in Theorem 1 of $[8,9]$ for the FLD-based system,

(iv) $\Psi(0)=1+\sum_{j=1}^{\bar{J}} P_{j}(\alpha)+(k /(1-p)) \sum_{l=1}^{M} c_{l}$ with $c_{l}$, $l=1, \ldots, M$, as in Theorem 2 of $[8,9]$ for the FFLDbased system.

Proof. Recall the specific form of the fractional Laguerre filters (10) incorporating the cascade combination of the firstorder filters $G_{L}^{f}\left(q^{-1}\right)$ and $\left[G_{R}^{f}\left(q^{-1}\right)-P\right]$, whose poles are just $\lambda_{1}^{f}=P-1$, which is the (only) eigenvalue in the corresponding (scalar) state space model as in [30, 31]. Since the argument $\varphi$ of that real-valued eigenvalue is zero, we consider the stability contour involving the function $\Psi(\varphi)$ as in Theorem 7 of [30], but for $\varphi$ equal to zero. Now, for the FD-based system we have $\Psi(\varphi)$ under specification (i) of Theorem 3 , so that $\Psi(0)=0$ and the stability condition is just $P<1$. Since LD/CFLD are equivalent to FD $[8,9]$, we arrive at the same condition for the LD/CFLD-based systems. For the FFD-based system we have $\Psi(\varphi)$ under specification (ii) of Theorem 3, so $\Phi(0)=1+\sum_{j=1}^{\bar{J}} P_{j}(\alpha)$, thus ending up with condition (22). In a similar way, for the FLD and FFLD-based systems we have, respectively, $\Psi(0)=1+(k /(1-p)) \sum_{l=1}^{M} c_{l}$, with $c_{l}, l=1, \ldots, M$, as in Theorem 1 of $[8,9]$, and $\Psi(0)=$ $1+\sum_{j=1}^{\bar{J}} P_{j}(\alpha)+(k /(1-p)) \sum_{l=1}^{M} c_{l}$ with $c_{l}, l=1, \ldots, M$, as in Theorem 2 of $[8,9]$, leading again to condition (22). Finally, the cascade combination of the considered filters is asymptotically stable if and only if all the particular filters are asymptotically stable, which completes the proof.

It is rather surprising that the Laguerre-based FFD system can be asymptotically stable even for $P>1$ (and $\alpha<1$ ). This stability result yielded from (22) is a nice theoretical confirmation of our earlier surprising simulation outcome of [35]. In fact, with $\sum_{j=1}^{\bar{J}} P_{j}(\alpha)>-1$ under $\alpha \in(0,1)$ and finite $\bar{J}$, the pole $P$ can be admitted higher than unity. But on the other hand, for $\alpha \in(1,2)$ the term $\sum_{j=1}^{\bar{J}} P_{j}(\alpha)$ can be lower than -1 so that even for $P<1$ the FFD system may be unstable, this being yet another nice stability result confirming our earlier surprising simulation observations.

Out of a plethora of simulations runs, we firstly present two selected examples.

Example 10. Consider the FFD-based fractional Laguerre system with $\bar{J}=20$ and two different values of $\alpha=0.5$ and $\alpha=1.5$. It can be concluded from Theorem 9 that for $\alpha=0.5$ the system is asymptotically stable for $P<1.12537$. On the other hand, for $\alpha=1.5$ the system is unstable for $P>0.99678$.

In a similar way, the FLD/FFLD-based fractional Laguerre systems can be stable even for $P>1$, which is illustrated in the following. 


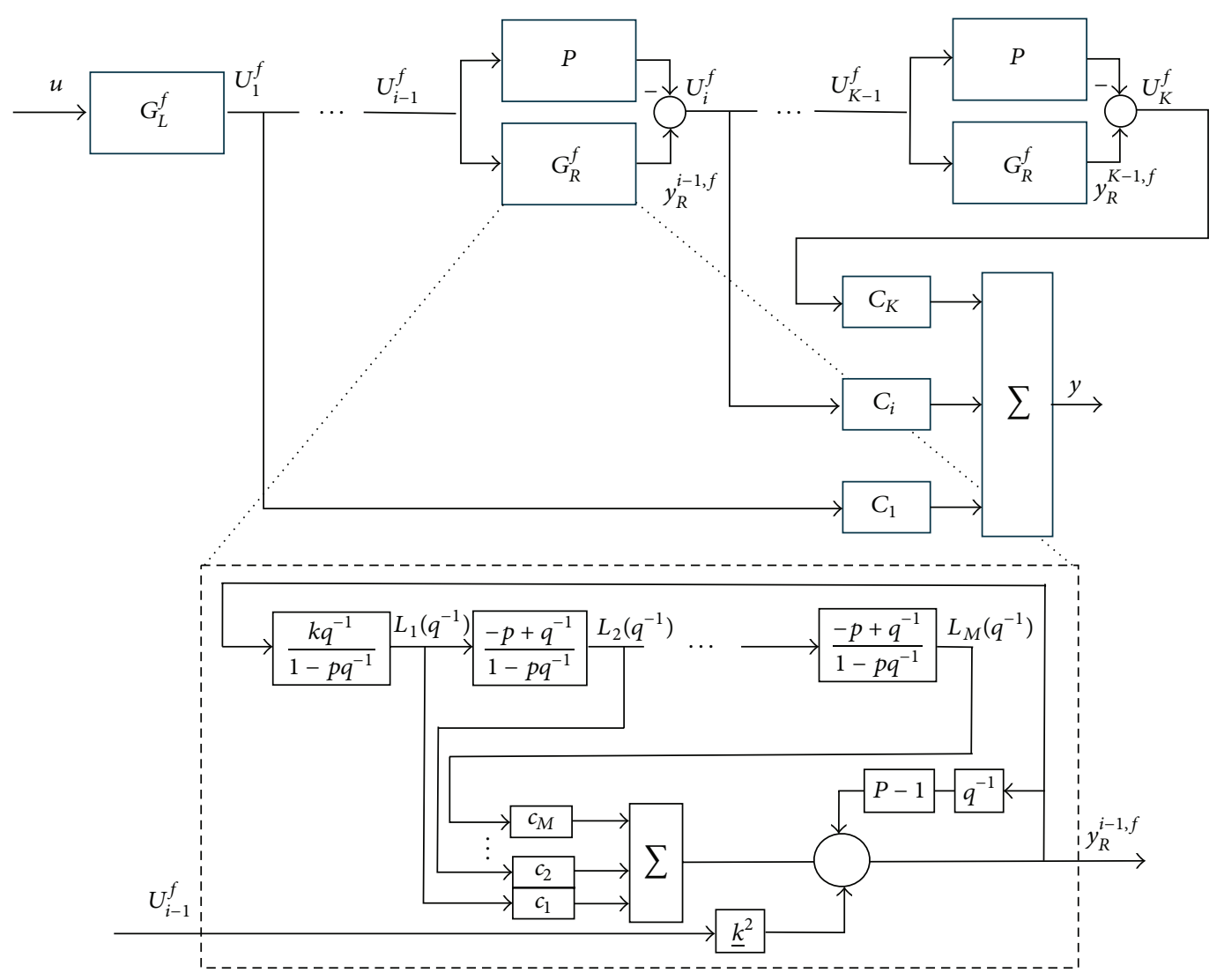

FIGURE 1: Block diagram of output calculation process for the FLD-based Laguerre model.

Example 11. Consider FLD/FFLD-based Laguerre systems with $P=1.005$. Let $M=10$ and $p=p_{\text {opt }}=0.58206$ in the FLD-based system and $\bar{J}=5, M=10$, and $p=p_{\text {opt }}=$ 0.91094 in the FFLD-based one. For the FLD-based Laguerre system we have $\Psi(0)=0.0235$ and for the FFLD-based one we have $\Psi(0)=6.94 e-3$, so in both cases condition (22) is satisfied and the two systems are asymptotically stable.

Remark 12. Note that the "closedness" of $\Psi(0)$ to zero is an excellent indicator of the quality of the specific approximator FFD/FLD/FFLD to FD/LD/CFLD.

Remark 13. In order to account for the sampling period $T$ when transferring from the continuous-time derivative to FD/LD/CFLD/FFD/FLD/FFLD, in the stability analysis for the fractional Laguerre system we replace (22) with

$$
T^{\alpha}(P-1)<\Psi(0), \quad \forall \alpha \in(0,2) \wedge P>0
$$

with $\Psi(0)$ as in Theorem 9 .

Remark 14. It is interesting that the stability condition for fractional-order Laguerre systems based on the FD difference (and equivalently, LD and CFLD ones $[8,9]$ ), that is, $P<1$, is identical with that for the regular integer-order Laguerre system. Also note that in this case $(\Psi(0)=0)$ the stability condition is, rather surprisingly, independent of both the sampling period $T$ and the order $\alpha$. In contrast, criterion (23) for the FFD/FLD/FFLD-based fractional Laguerre systems is always dependent on both $T$ and $\alpha$, and this is because $\Psi(0) \neq 0$ in that case.

\section{Stability Preservation Analysis}

The stability preservation issue for fractional-order systems under direct discretization methods has been considered in [36]. The problem reduces to the provision of a stable discretized system approximating its stable continuous-time original. Here we present our stability preservation results for the FFD/FLD/FFLD-based Laguerre systems.

Theorem 15. Consider the (discretized) FFD/FLD/FFLDbased Laguerre system as in Theorem 3, under respective specifications (ii), (iii), and (iv). Assume that

(1) $\Psi(0)>0$ and $P<1$,

(2) $\Psi(0)>0$ and $P>1$,

(3) $\Psi(0)<0$ and $P<1$, or

(4) $\Psi(0)<0$ and $P>1$.

Then, respectively,

(1) the system is asymptotically stable regardless of the sampling period,

(2) the system is asymptotically stable if and only if $T<$ $[\Psi(0) /(P-1)]^{1 / \alpha}$, 
TABLE 1: Stability results for FFD/FLD/FFLD-based fractional-order Laguerre systems.

\begin{tabular}{|c|c|c|c|c|c|}
\hline Approximation & $\alpha$ & $T$ & $P$ & Condition & Comment \\
\hline $\mathrm{FFD}(\bar{J}=100)$ & 0.7 & 0.5 & $P=0.99$ & Stable & Stable $^{1}$ \\
\hline $\operatorname{FFD}(\bar{J}=100)$ & 0.5 & 0.5 & $P=1.02$ & $T<7.94$ & Stable $^{2}$ \\
\hline $\operatorname{FFD}(\bar{J}=100)$ & 1.2 & 0.5 & $P=0.99$ & $T>0.107$ & Stable $^{3}$ \\
\hline $\operatorname{FFD}(\bar{J}=100)$ & 1.5 & 0.5 & $P=1.02$ & Unstable & Unstable $^{4}$ \\
\hline $\operatorname{FLD}(M=20, p=0.765)$ & 0.5 & 5 & $P=1.02$ & $T<4.90$ & Unstable $^{2}$ \\
\hline $\operatorname{FLD}(M=20, p=0.577)$ & 1.2 & 1 & $P=0.995$ & $T>0.248$ & Stable ${ }^{3}$ \\
\hline $\operatorname{FFLD}(\bar{J}=10, M=20, p=0.959)$ & 0.8 & 0.8 & $P=1.001$ & $T<0.805$ & Stable $^{2}$ \\
\hline $\operatorname{FFLD}(\bar{J}=10, M=20, p=0.933)$ & 1.3 & 0.1 & $P=0.999$ & $T>0.104$ & Unstable $^{3}$ \\
\hline
\end{tabular}

(3) the system is asymptotically stable if and only if $T>$ $[\Psi(0) /(P-1)]^{1 / \alpha}$,

(4) the system is unstable regardless of the sampling period.

Proof. Immediate from condition (23).

Some remarks are due, now. Firstly, the results for variants 1 and 3 of Theorem 15 are quite surprising. Secondly, we have verified all the variants of Theorem 15 in a plethora of simulation runs, the selected examples of which are tabulated. Table 1 collects the results, with the particular variants of Theorem 15 superindexed in the Comment column. The results of Table 1 are self-explanatory.

\section{Parameter Estimation for Fractional Laguerre Filter}

When output (9) (or (20)) of the fractional Laguerre filter is corrupted with noise and the order $\alpha$ is known, the model output $\hat{y}(t)$ can be described in a linear regression fashion $\hat{y}(t)=\varphi^{T}(t) \Theta$, with $\varphi^{T}(t)=\left[U_{1}^{f}(t), U_{2}^{f}(t), \ldots, U_{K}^{f}(t)\right]$ and the unknown parameters $\Theta^{T}=\left[C_{1}, C_{2}, \ldots, C_{K}\right]$ estimated analytically, for example, (R)LS method. When $\alpha$ is unknown the parameter vector $\Theta^{T}=\left[C_{1}, C_{2}, \ldots, C_{K}, \alpha\right]$ can be estimated numerically using, for example, LS or, preferably, GA procedures.

Example 16. An FD-based discrete-time state space system $\left\{A_{f}, B, C, \alpha_{s}\right\}$, with its fractional (commensurate) order $\alpha_{s}=$ 0.85 and

$$
\begin{aligned}
A_{f} & =\left[\begin{array}{cccc}
2.37 & -4.3849 & 2.602023 & -0.5886251 \\
1 & -1 & 0 & 0 \\
0 & 1 & -1 & 0 \\
0 & 0 & 1 & -1
\end{array}\right], \\
B^{T} & =\left[\begin{array}{llll}
1 & 0 & 0 & 0
\end{array}\right], \\
C & =\left[\begin{array}{llll}
1 & -1.8 & 0.85 & 0
\end{array}\right]
\end{aligned}
$$

and the output white noise of zero mean and standard deviation $\sigma_{e}=0.0001$, is modeled by means of the FFD$(\bar{J}=500)$, FLD- $(M=35)$, and FFLD- $(\bar{J}=20, M=$ 25) based fractional Laguerre models with optimally selected
TABLE 2: Mean square prediction errors for the analyzed models; Example 16.

\begin{tabular}{lc}
\hline Models & MSPE \\
\hline FFD-based Laguerre model & 12.71 \\
FLD-based Laguerre model & 9.80 \\
FFLD-based Laguerre model & 4.88 \\
\hline
\end{tabular}

TABLE 3: Standard deviations of frequency responses for the analyzed models; Example 16.

\begin{tabular}{lcc}
\hline Models & Magnitude error [dB] & Phase error [DEG] \\
\hline FFD-based Laguerre & 0.1002 & 0.707 \\
FLD-based Laguerre & 0.0938 & 0.777 \\
FFLD-based Laguerre & 0.0851 & 0.608 \\
\hline
\end{tabular}

$\alpha=0.903$ (via a combined LS/GA algorithm) and $P=0.87$. The $K$ parameter is selected (heuristically) to be equal to 16 and $C_{i}, i=1, \ldots, K$, parameters are estimated by the RLS method. The input is a discrete-time zero mean unityvariance Gaussian random signal. The MSPEs for the outputs of the three Laguerre-based models with respect to that for the FD-based state space system are presented in Table 2.

Table 2 shows that the best performance is obtained for the FFLD-based Laguerre model. In this case, the FFLDbased fractional Laguerre model with 45 parameters gives essentially lower MSPE than the FFD-based one with 500 parameters. This shows that the FFLD is a very good approximation to FD. Also, a simple FLD-based fractional Laguerre model, with 35 parameters only, gives satisfactory results.

The frequency responses of the actual system and (hardly distinguishable) FFD/FLD/FFLD-based fractional Laguerre models are presented in Figure 2. Table 3 presents standard deviations of the magnitude and phase of the frequency responses for the FFD/FLD/FFLD-based fractional Laguerre models with respect to the FD-based system.

Remark 17. It is interesting that fractional Laguerre-based models may have their fractional orders $(\alpha)$ different from those for fractional state space originals $\left(\alpha_{s}\right)$; see Example 16. The discrepancy is related to the spectrum of eigenvalues of $A_{f}$. Specifically, for the "slightly" oscillatory eigenvalues as in Example $16\left(\lambda_{1,2}=-0.15 \pm 0.38 i\right)$ we have $\alpha=0.903$ 

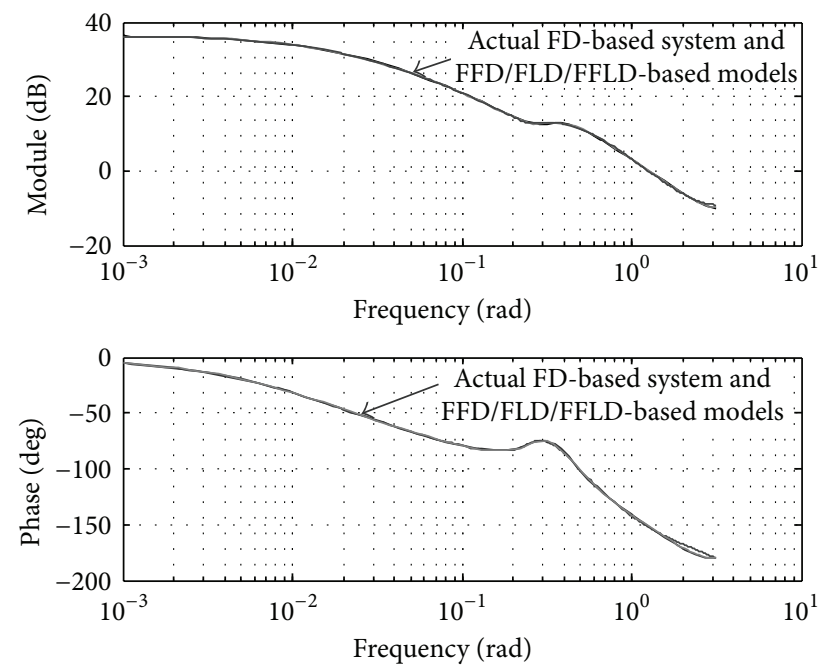

FIGURE 2: Bode plots of the actual system versus FFD/FLD/FFLDbased fractional Laguerre models; Example 16.

closer to unity than the original $\alpha_{s}=0.85$. Also, "strong" oscillatory fractional behaviors may be Laguerre modeled with $\alpha \in(1,2)$; compare Remark 8 .

\section{The Reorthonormalization Issue}

A side effect of factorizations (3) or (10) is that the orthonormality property is lost for fractional Laguerre systems; that is, the functions $L_{i}^{f}\left(z^{-1}\right)=G_{L}^{f}\left(z^{-1}\right)\left[G_{R}^{f}\left(z^{-1}\right)-P\right]^{i-1}, i=$ $1, \ldots, K$, are no longer orthonormal. However, the fractional Laguerre filters can be easily reorthonormalized using the standard procedures $[18,37]$, with the results illustrated with Example 18.

Example 18. Consider the system as in Example 16 and two FFLD-based fractional Laguerre models with parameters as in Example 16 with (a) nonorthonormalized and (b) reorthonormalized Laguerre filters. The model parameters are estimated using the RLS method.

The simulation experiments show that both models give quite the same MSPE errors equal to some 4.88. So, the reorthonormalization process may not improve the model accuracy. However, it is well known that the reorthonormalization process improves conditioning of the covariance matrix in the RLS estimation process. This is shown in Figure 3, which presents the condition numbers of the covariance matrix $P(t)$ as a function of time $t$ for both orthonormalized and nonorthonormalized fractional Laguerre filters.

\section{Conclusion}

This paper has presented a series of new results in modeling and analysis of discrete-time fractional-order LTI SISO systems using Laguerre filters. In the "lower" modeling layer described in $[8,9]$, the Grünwald-Letnikov fractionalorder difference has been effectively approximated by the Laguerre filters. This paper has produced new results related to modeling and analysis of the "upper" system dynamics

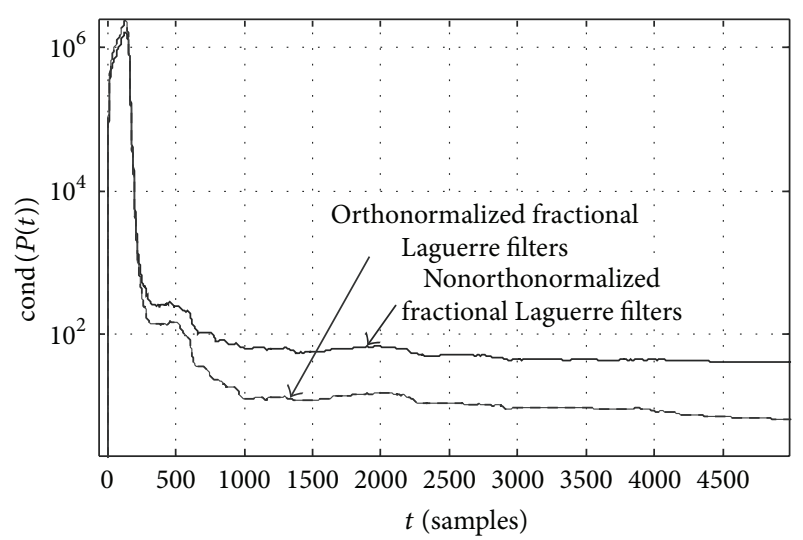

FIgURE 3: Conditioning of the covariance matrix versus time; Example 18.

layer. Firstly, new "fractional-order discrete-time Laguerre filters" have been shown to efficiently model discrete-time fractional-order LTI SISO systems. Secondly, a new analytical stability criterion has been announced for discretetime fractional-order Laguerre systems. Thirdly, new results on stability preservation for discretized fractional-order Laguerre systems have been derived. New achievements of the paper have been illustrated with simulation examples. Our current and future research works are directed to predictive/adaptive control of Laguerre-based fractional-order discrete-time MIMO systems, both linear and nonlinear ones.

\section{Nomenclature}

CFLD: Combined fractional/Laguerre-based difference

FD: Grünwald-Letnikov fractional-order difference

FFD: Finite fractional difference

FFLD: Finite (combined) fractional/Laguerre-based difference

FLD: Finite Laguerre-based difference

GL: Grünwald-Letnikov

LD: Laguerre-based difference

OBF: Orthonormal basis functions.

\section{Competing Interests}

The authors declare that they have no competing interests.

\section{References}

[1] A. Dubravić and Z. Šehić, "Using orthonormal functions in model predictive control," Tehnicki Vjesnik, vol. 19, no. 3, pp. 513-520, 2012.

[2] P. S. C. Heuberger, P. M. J. Van den Hof, and B. Wahlberg, Modeling and Identification with Rational Orthogonal Basis Functions, Springer, London, UK, 2005.

[3] B. Ninness, H. Hjalmarsson, and F. Gustafsson, "The fundamental role of general orthonormal bases in system identification," 
IEEE Transactions on Automatic Control, vol. 44, no. 7, pp. 13841406, 1999.

[4] O. Mykhailenko, "Cone crusher model identification using block-oriented systems with orthonormal basis functions," International Journal of Control Theory and Computer Modeling, vol. 4, no. 3, pp. 1-8, 2014.

[5] A. Wills, T. B. Schön, L. Ljung, and B. Ninness, "Identification of Hammerstein-Wiener models," Automatica, vol. 49, no. 1, pp. 70-81, 2013.

[6] I. Area, J. D. Djida, J. Losada, and J. J. Nieto, "On fractional orthonormal polynomials of a discrete variable," Discrete Dynamics in Nature and Society, vol. 2015, Article ID 141325, 7 pages, 2015.

[7] G. Maione, "On the Laguerre rational approximation to fractional discrete derivative and integral operators," IEEE Transactions on Automatic Control, vol. 58, no. 6, pp. 1579-1585, 2013.

[8] R. Stanisławski, "New Laguerre filter approximators to the Grünwald-Letnikov fractional difference," Mathematical Problems in Engineering, vol. 2012, Article ID 732917, 21 pages, 2012.

[9] R. Stanisławski, K. J. Latawiec, and M. Łukaniszyn, "A comparative analysis of Laguerre-based approximators to the GrünwaldLetnikov fractional-order difference," Mathematical Problems in Engineering, vol. 2015, Article ID 512104, 10 pages, 2015.

[10] Y. Q. Chen, I. Petras, and D. Xue, "Fractional order controla tutorial," in Proceedings of the American Control Conference (ACC '09), pp. 1397-1411, St. Louis, Mo, USA, June 2009.

[11] W. Krajewski and U. Viaro, "A method for the integer-order approximation of fractional-order systems," Journal of the Franklin Institute, vol. 351, no. 1, pp. 555-564, 2014.

[12] B. T. Krishna, "Studies on fractional order differentiators and integrators: a survey," Signal Processing, vol. 91, no. 3, pp. 386426, 2011.

[13] M. Rachid, B. Maamar, and D. Said, "Comparison between two approximation methods of state space fractional systems," Signal Processing, vol. 91, no. 3, pp. 461-469, 2011.

[14] C. Monje, Y. Chen, B. Vinagre, D. Xue, and V. Feliu, Fractionalorder Systems and Controls: Fundamentals and Applications, Series on Advances in Industrial Control, Springer, London, UK, 2010.

[15] D. Mozyrska, "Multiparameter fractional difference linear control systems," Discrete Dynamics in Nature and Society, vol. 2014, Article ID 183782, 8 pages, 2014.

[16] I. Podlubny, Fractional Differential Equations, vol. 198 of Mathematics in Science and Engineering, Academic Press, Orlando, Fla, USA, 1999.

[17] H. Sheng, Y. Q. Chen, and T. S. Qiu, Fractional Processes and Fractional-Order Signal Processing, Springer, New York, NY, USA, 2012.

[18] M. Aoun, R. Malti, F. Levron, and A. Oustaloup, "Synthesis of fractional Laguerre basis for system approximation," Automatica, vol. 43, no. 9, pp. 1640-1648, 2007.

[19] R. Stanisławski, K. J. Latawiec, W. P. Hunek, and M. Zukaniszyn, "Laguerre-based modeling of fractional-order LTI SISO systems," in Proceedings of the 18th International Conference on Methods and Models in Automation and Robotics (MMAR '13), pp. 610-615, Miedzyzdroje, Poland, August 2013.

[20] R. Stanisławski and K. J. Latawiec, "Normalized finite fractional differences: computational and accuracy breakthroughs," International Journal of Applied Mathematics and Computer Science, vol. 22, no. 4, pp. 907-919, 2012.
[21] P. Ostalczyk, "Equivalent descriptions of a discrete-time fractional-order linear system and its stability domains," International Journal of Applied Mathematics and Computer Science, vol. 22, no. 3, pp. 533-538, 2012.

[22] D. Mozyrska and M. Wyrwas, "The $\mathscr{Z}$-transform method and delta type fractional difference operators," Discrete Dynamics in Nature and Society, vol. 2015, Article ID 852734, 12 pages, 2015.

[23] S. Guermah, S. Djennoune, and M. Bettayeb, "A new approach for stability analysis of linear discrete-time fractional-order systems," in New Trends in Nanotechnology and Fractional Calculus Applications, pp. 151-162, Springer, Berlin, Germany, 2010.

[24] I. Kheirizad, M. S. Tavazoei, and A. Jalali, "Stability criteria for a class of fractional order systems," Nonlinear Dynamics, vol. 61, no. 1-2, pp. 153-161, 2010.

[25] C. Li and Z. Zhao, "Asymptotical stability analysis of linear fractional differential systems," Journal of Shanghai University (English Edition), vol. 13, no. 3, pp. 197-206, 2009.

[26] I. Petráš, "Stability of fractional-order systems with rational orders: a survey," Fractional Calculus \& Applied Analysis, vol. 12, no. 3, pp. 269-298, 2009.

[27] J. Sabatier, M. Moze, and C. Farges, "On stability of fractional order systems," in Proceedings of the 3rd IFAC Workshop on Fractional Differentiation and Its Applications (FDA'08), Ankara, Turkey, November 2008.

[28] S. B. Stojanovic and D. L. Debeljkovic, "Simple stability conditions of linear discrete time systems with multiple delay," Serbian Journal of Electrical Engineering, vol. 7, no. 1, pp. 69-79, 2010.

[29] D. Matignon, "Stability results for fractional differential equations with applications to control processing," in Proceedings of the Computational Engineering in Systems and Applications Multiconference, vol. 2, pp. 963-968, Lille, France, 1996.

[30] R. Stanisławski and K. J. Latawiec, "Stability analysis for discrete-time fractional-order LTI statespace systems. Part I: new necessary and sufficient conditions for asymptotic stability," Bulletin of the Polish Academy of Sciences, Technical Sciences, vol. 61, no. 2, pp. 353-361, 2013.

[31] R. Stanisławski and K. J. Latawiec, "Stability analysis for discrete-time fractional-order LTI statespace systems. Part II: new stability criterion for FD-based systems," Bulletin of the Polish Academy of Sciences, Technical Sciences, vol. 61, no. 2, pp. 362-370, 2013.

[32] C. Boukis, D. P. Mandic, A. G. Constantinides, and L. C. Polymenakos, "A novel algorithm for the adaptation of the pole of Laguerre filters," IEEE Signal Processing Letters, vol. 13, no. 7, pp. 429-432, 2006.

[33] S. T. Oliveira, "Optimal pole conditions for Laguerre and twoparameter Kautz models: a survey of known results," in Proceedings of the 12th IFAC Symposium on System Identification (SYSID '00), pp. 457-462, Santa Barbara, Calif, June 2000.

[34] Q. Chen, W. Mai, L. Zhang, and W. Mi, "System identification by discrete rational atoms," Automatica, vol. 56, pp. 53-59, 2015.

[35] R. Stanisławski, "Identification of open-loop stable linear systems using fractional orthonormal basis functions," in Proceedings of the 14th International Conference on Methods and Models in Automation and Robotics, pp. 935-985, Miedzyzdroje, Poland, 2009.

[36] M. Siami, M. S. Tavazoei, and M. Haeri, "Stability preservation analysis in direct discretization of fractional order transfer functions," Signal Processing, vol. 91, no. 3, pp. 508-512, 2011. 
[37] P. M. Van den Hof, P. S. Heuberger, and J. Bokor, "System identification with generalized orthonormal basis functions," Automatica, vol. 31, no. 12, pp. 1821-1834, 1995. 


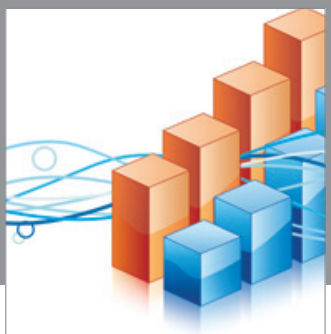

Advances in

Operations Research

vatem alat4

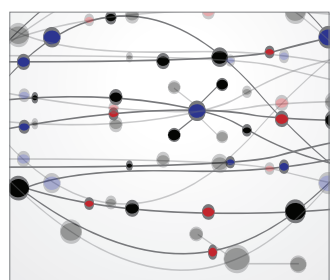

\section{The Scientific} World Journal
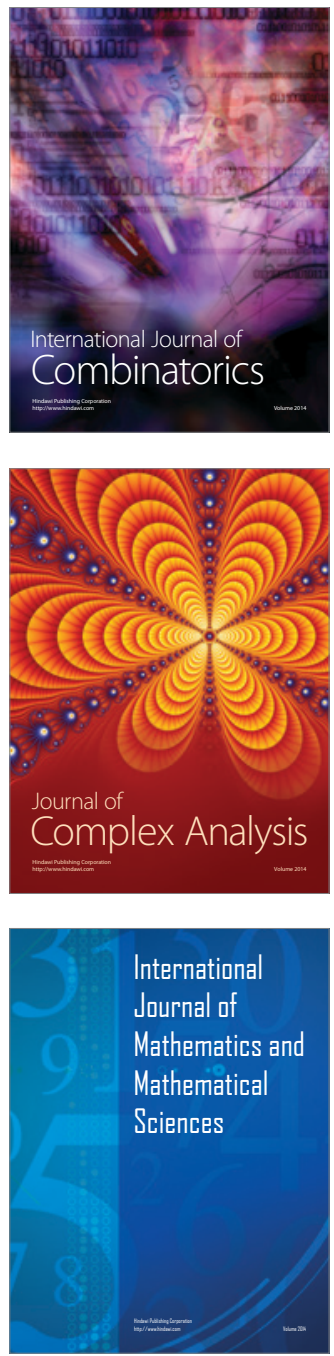
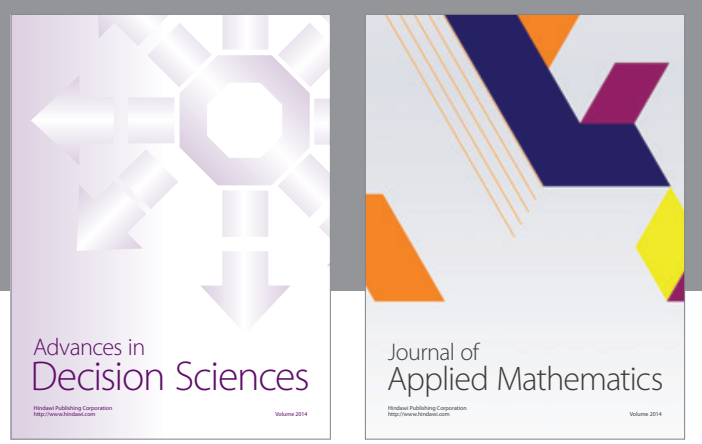

Algebra

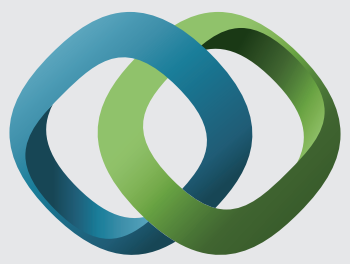

\section{Hindawi}

Submit your manuscripts at

http://www.hindawi.com
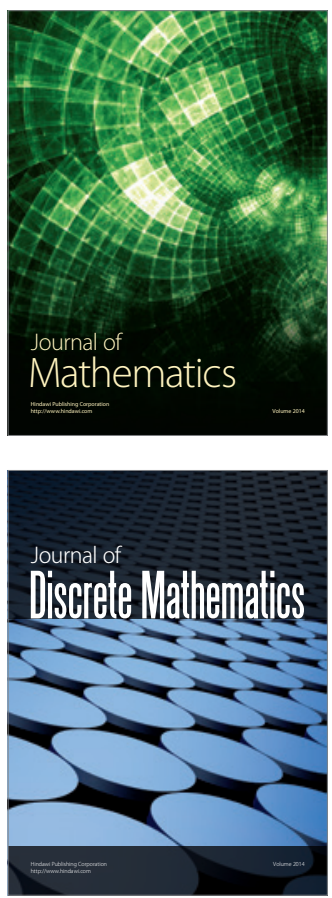

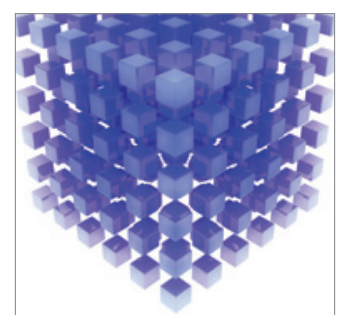

Mathematical Problems in Engineering
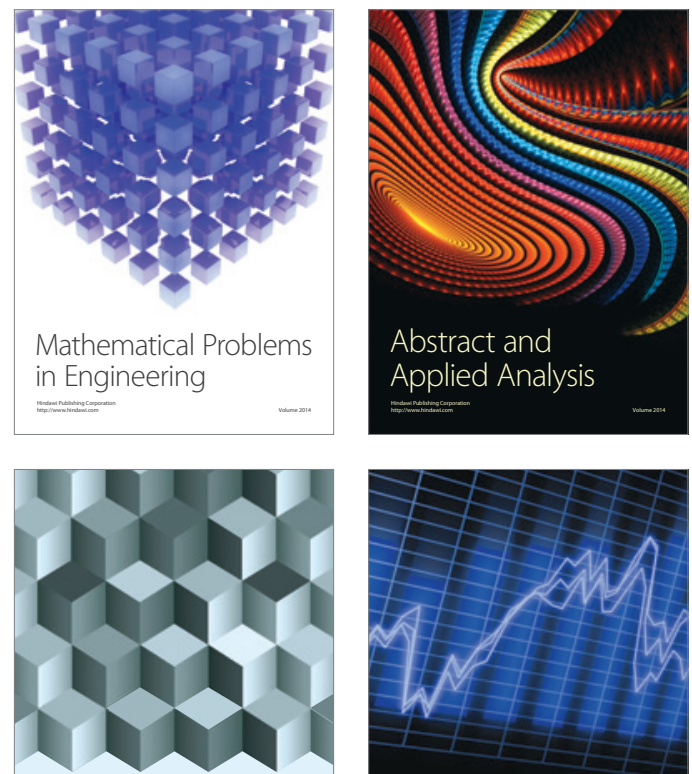

Journal of

Function Spaces

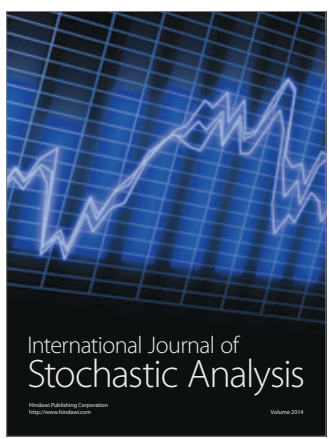

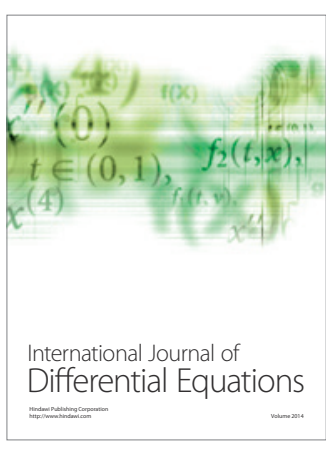
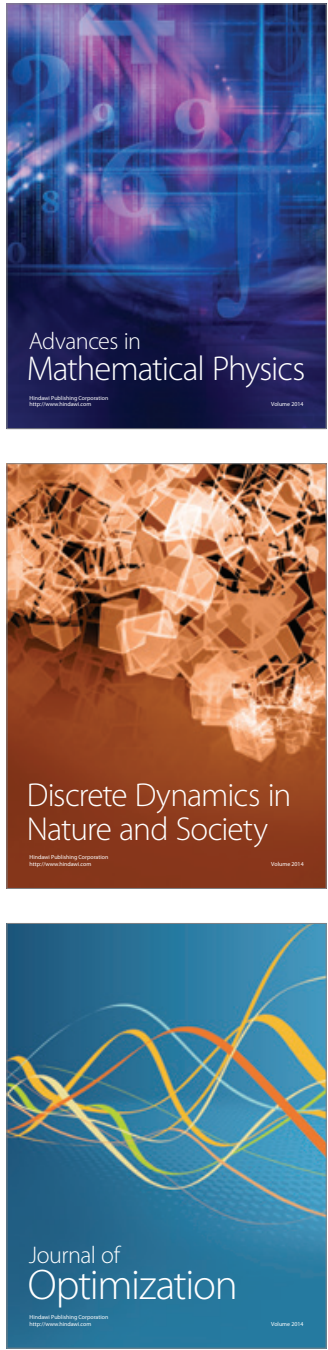